\title{
Talento humano y desarrollo organizacional en las microempresas turísticas del Valle del Mantaro
}

\author{
Human talent and organizational development in the micro-enterprises \\ of the Mantaro Valley
}

Isac F. Espinoza M. ${ }^{1}$, Haydee Segovia H. ${ }^{1}$ \& Henry F. Espinoza S. ${ }^{1}$

Facultad de Ciencias de la Administración de la Universidad Nacional del Centro del Perú.

Email: espinozaisac@yahoo.es

\section{RESUMEN}

Basado en los resultados de la investigación titulada; Competitividad de la micro y pequeña empresa turística del valle del Mantaro (Espinoza Montes \& Espinoza Segovia, 2012), donde se concluye que; Los recursos humanos, constituyen el factor más débil en las Mypes (1.37), se inicia esta investigación postulando que: El talento humano determina el desarrollo organizacional en microempresas turísticas del Valle del Mantaro en medida significativa, manteniendo un estado de atraso. Utilizando el método deductivo y el analítico sintético, las técnicas de la encuesta, la estadística descriptiva (media) e inferencial, el estudio explica en qué medida el talento humano se relaciona con el desarrollo organizacional en microempresas turísticas del valle del Mantaro. Con un nivel de significancia de $5 \%$, los resultados indican que la variable Talento Humano, tiene poder explicativo y es significativo para explicar Desarrollo Organizacional. Los resultados también indican que el talento humano, está determinada por sólo dos dimensiones más relevantes: El compromiso y la acción del personal. Es así que se contrasta que el talento humano se relaciona con el desarrollo organizacional en microempresas turísticas del Valle del Mantaro en medida significativa, manteniendo un estado de atraso.

\begin{abstract}
Based on the results of the research entitled; competitiveness of micro and small tourism businesses in the Mantaro Valley (Espinoza Montes \& Espinoza Segovia, 2012), which concludes that, Human resources constitute the weakest factor in MSEs ( 1.37 ), this research begins by postulating that: human talent determines organizational development in tourism micro Mantaro Valley in significant measure, maintaining a state of backwardness. Using the deductive inference and the synthetic analytical, technical survey, the (mean) statistics and the study aims to explain how human talent determines organizational development in tourism micro Mantaro valley. Results with a significance level of 5 $\%$, indicate that the variable Talent, has significant explanatory power to explain and Organizational Development. According to the results the human talent variable is explained by only two relevant dimensions which are: staff commitment and action. Thus, we can test that determines human talent organizational development in tourist micro Mantaro Valley in significant measure, maintaining a state of backwardness.
\end{abstract}

Palabras clave | Key words:

talento humano, desarrollo organizacional. human talent, organizational development. 


\section{INTRODUCCIÓN}

LEI Valle del Mantaro, por lo descrito por varios autores y la percepción de muchos especialistas, tiene un alto potencial de desarrollo en el turismo, sin embargo los organismos a cargo del desarrollo del sector gestionan deficientemente dicha actividad. Uno de los problemas cruciales, involucra a los trabajadores. En ese marco, la investigación parte de la siguiente interrogante; $\dot{E}$ En qué medida el talento humano determina el desarrollo organizacional en microempresas turísticas del valle del Mantaro?. De acuerdo a observaciones previas, el análisis y razonamiento deductivo e inductivo, se postula que; El talento humano determina el desarrollo organizacional en microempresas turísticas del valle del Mantaro en medida significativa, manteniendo un estado de atraso.

Experiencias en el mundo explican las grandes potencialidades y ventajas económicas y medioambientales que se puede obtener del sector, lo que repercutiría en el desarrollo económico de estas unidades en el marco de las políticas públicas del gobierno de crear empleo decente para la población; y es en mérito a ello que la investigación pretende evaluar la incidencia del talento humano en el desarrollo organizacional en las microempresas turísticas del valle del Mantaro.

Al respecto, la comunidad científica acepta que un talento siempre es un tipo especial de persona y no siempre toda persona es un talento, la persona debe poseer algún diferencial competitivo que la valore como conocimiento, habilidad, juicio y actitud. (Chiavenato, 2009). El talento requiere de tres ingredientes básicos: capacidades, compromiso y acción. (Jericó, 2001): (1) Capacidades, que son los conocimientos, habilidades y competencias o actitudes, (2) compromiso. Que se constituye en el motor para que el profesional aporte lo máximo posible y no marche a otra compañía y (3) Acción, que significa velocidad e innovación constante. Puesto que no puede detenerse la evolución tecnológica lo mejor es posicionarse en primera línea y hacerlo antes que otro.

Del mismo modo, el D.O. tiene diferentes significados para diferentes personas. No existe una definición que complazca a todos. Diversos autores y profesionistas han presentado diferentes definiciones. (De Faria, 2004). A partir del estado de la cuestión, el estudio tendrá como an- tecedente fundamental el trabajo realizado por Friedlander y Brown (1974), que presenta el DO como una metodología para facilitar cambios y desarrollo en: personas, tecnologías, procesos y estructuras organizacionales y DO como resultado en términos de; personas, tecnologías, procesos y estructuras organizacionales.

En ese orden de ideas, la investigación planteó como objetivo; determinar en qué medida el talento humano se relaciona con el desarrollo organizacional en microempresas turísticas del valle del Mantaro, para proponer programas de intervención.

\section{MATERIALES Y MÉTODOS}

La investigación se realizó utilizando el Método Científico. Los métodos de inducción y deducción y el análisis y síntesis se utilizaron en la construcción hipotética y teórica y en la actividad operativa de la investigación respectivamente.

Por tratarse de una investigación no experimental, aplicado, ex post facto, de corte transversal, como estrategia de constatación, es decir de recolección de datos, se utilizó la técnica de encuesta, que fue complementada con la estrategia de triangulación con utilización de data secundaria y entrevistas en profundidad.

En el proceso de determinación de la muestra y en el procesamiento de datos se utilizó el software Excel con un nivel de significancia de 0.05 y nivel de confianza de 95\%, para población de microempresas desconocida y probabilidad de buena gestión del talento humano de 0.10 $(n=139 * 2$ trabajadores por empresa $=278$ trabajadores).

Para la prueba de hipótesis se utilizó el análisis de regresión múltiple, ANOVA, F y la prueba † Student, por carencia de los datos respecto de la varianza de la población.

\section{RESULTADOS}

Como se observa en la figura 2, las subvariables de talento humano estudiadas microempresas turísticas del valle del Mantaro no alcanzan niveles aceptables, al contrario se puede decir que el personal de las microempresas turísticas del valle del Mantaro en general no se constituyen como talentos, pues tienen un nivel mínimo de conocimientos, actitudes, de capaci- 
dad para toma de decisiones, sumándose a ello el alto grado de rotación externa y absentismo. En las subvariables mencionadas, alcanzan un puntaje medio inferior de 2. Sólo en habilidades y actitudes obtienen puntajes superiores superiores a 2 .

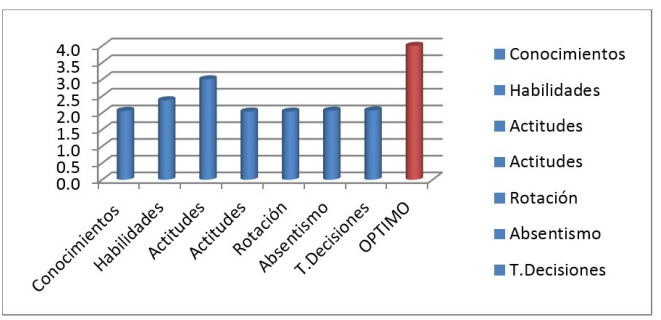

Figura 2. Subvariables de talento humano en microempresas turísticas del Valle del Mantaro.

Como se observa en la figura 2, las subvariables de desarrollo organizacional estudiadas en microempresas turísticas del valle del Mantaro tampoco alcanzan niveles aceptables.

Es así que los niveles alcanzados en general son inferiores a la anterior variable. Existe un mínimo desarrollo tecnológico y un mínimo desarrollo en las estructuras organizacionales (alcanzando un puntaje de 1 de un total de 4). Mejor pero no satisfactorio se encuentran el desarrollo personal y desarrollo de procesos, alcanzando un puntaje de 2 de un total de 4 .

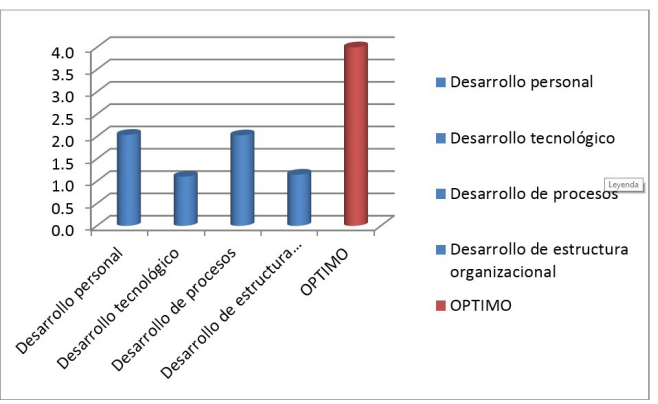

Figura 3. Subvariables de desarrollo organizacional

En términos generales el talento humano; tiene capacidades superiores ligeramente a la media, pero no se encuentra comprometido con las empresas y sus acciones son muy insuficientes para pretender el impulso de estas empresas.

Esta situación se hace más preocupante cuando se compara con el desarrollo organizacional, nuestras microempresas turísticas del Valle del
Mantaro, tienen un bajo nivel de desarrollo organizacional.

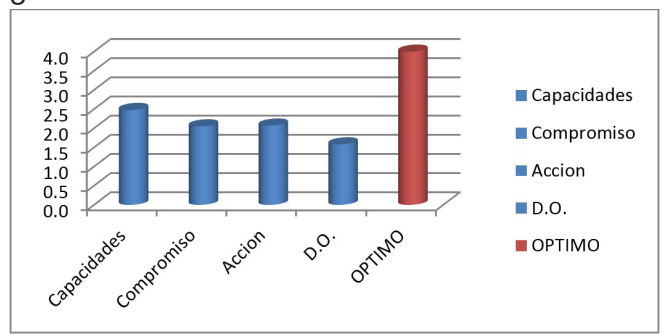

Figura 3. Dimensiones de talento humano y desarrollo organizacional

La hipótesis general de investigación postuló que: El talento humano se relaciona con el desarrollo organizacional en microempresas turísticas del valle del Mantaro en medida significativa, manteniendo un estado de atraso. A partir de la hipótesis de investigación se formuló las hipótesis estadísticas siguientes:

Ho: El talento humano no se relaciona con el desarrollo organizacional en microempresas turísticas del Valle del Mantaro en medida significativa.

Ha: El talento humano determina el desarrollo organizacional en microempresas turísticas del Valle del Mantaro en medida significativa.

Tabla 4

Estadísticas de la regresión

Coeficiente de correlación múltiple

0.876

Coeficiente de determinación $\mathrm{R}^{\wedge} 2$

0.768

$\mathrm{R}^{\wedge} 2$ ajustado

0.766

Error típico

Observaciones

278.000

Tabla 5

Análisis de varianza

\begin{tabular}{|c|c|c|c|c|c|}
\hline & $\begin{array}{l}\text { Grados de } \\
\text { libertad }\end{array}$ & $\begin{array}{c}\text { Suma de } \\
\text { cuadrados }\end{array}$ & $\begin{array}{c}\text { Promedio } \\
\text { de los } \\
\text { cuadrados }\end{array}$ & $F$ & $\begin{array}{c}\text { Valor crítico } \\
\text { de F }\end{array}$ \\
\hline Regresión & 2 & 24.980 & 12.490 & 454.533 & 0.000 \\
\hline Residuos & 275 & 7.557 & 0.027 & & \\
\hline Total & 277 & 32.536 & & & \\
\hline
\end{tabular}




\begin{tabular}{|c|c|c|c|c|c|c|c|c|}
\hline & Coeficienties & $\begin{array}{l}\text { Error } \\
\text { típico }\end{array}$ & $\begin{array}{c}\text { Estadisico } \\
1\end{array}$ & Probabililad & $\begin{array}{l}\text { Inferior } \\
\text { 95\% }\end{array}$ & $\begin{array}{l}\text { Suppior } \\
95 \%\end{array}$ & $\begin{array}{l}\text { Interior } \\
95.0 \%\end{array}$ & $\begin{array}{l}\text { Superior } \\
95.0 \%\end{array}$ \\
\hline Interceopción & .1033 & 0.088 & -11.8007 & 0.000 & -1.206 & .0801 & -1.206 & .0 .861 \\
\hline VariableX1 & 1.015 & 0.057 & 177.24 & 0.000 & 0.903 & 1.128 & 0.003 & 1.128 \\
\hline Varable X2 & 0.257 & 0.050 & 5.136 & 0.000 & 0.159 & 0.356 & 0.159 & 0.356 \\
\hline
\end{tabular}

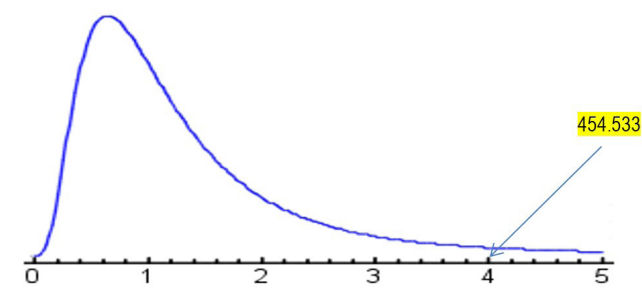

Figura 4. Contrastación de hipótesis general (F).

Con un nivel de significancia de $5 \%$ y nivel de confianza de 95\%, la variable Talento Humano, tiene poder explicativo y es significativo para explicar Desarrollo Organizacional. De acuerdo a los resultados la variable Desarrollo Organizacional, está explicada por sólo dos dimensiones más relevantes las cuales son: compromiso y acción del personal. Es así que se prueba que el Talento Humano determina el desarrollo organizacional en microempresas turísticas del Valle del Mantaro en medida significativa, manteniendo un estado de atraso.

\section{DISCUSIÓN}

De acuerdo a los resultados de la investigación, la variable Desarrollo Organizacional, está explicada por sólo dos dimensiones más relevantes las cuales son: compromiso y acción del personal. Es así que se puede contrastar que el talento humano determina el desarrollo organizacional en microempresas turísticas del Valle del Mantaro en medida significativa, manteniendo un estado de atraso.

Las subvariables, expresan coincidencias con los resultados encontrados por (Alcantara Napa, 2012), debido a que La mayoría de microempresas del distrito de Grocio Prado, provincia de Chincha ,2012, no invirtió en tecnología en su mayoría lo cual reflejó los bajos niveles de desarrollo organizacional por la modernidad y el creciente mundo globalizado por el paso del tiempo esto originó pérdidas debido a que ingresó nuevos clientes y no huno la capacidad por eso no atendió pedidos y perdió clientes En forma específica se ha encontrado que, el compromiso de los trabajadores incide directamente en el desarrollo organizacional en microempresas turísticas del Valle del Mantaro. A mayor compromiso, mayor desarrollo, e inversamente. Encontrando un bajo nivel de compromiso de los trabajadores.

Así mismo se ha encontrado que la acción de los trabajadores incide en el desarrollo organizacional en microempresas turísticas del Valle del Mantaro de manera directa. A mayor acción de los trabajadores, mayor desarrollo. Se ha encontrado poca iniciativa y acción de los trabajadores.

El estudio de investigación determino las siguientes conclusiones:

1. Con un nivel de significancia de $5 \%$ y nivel de confianza de 95\%, la variable Talento Humano, tiene poder explicativo y es significativo para explicar Desarrollo Organizacional. De acuerdo a los resultados la variable Desarrollo Organizacional, está explicada por sólo dos dimensiones más relevantes las cuales son: compromiso y acción del personal. Es así que se puede contrastar que el Talento Humano determina el Desarrollo Organizacional en microempresas turísticas del Valle del Mantaro en medida significativa, manteniendo un estado de atraso.

2. La hipótesis especifica uno postula que: las capacidades de los trabajadores inciden en el Desarrollo Organizacional en microempresas turísticas del Valle del Mantaro en gran magnitud. Con nivel de confianza de $95 \%$ y significancia de $5 \%(a=0.000)$, las capacidades de los trabajadores, no determina el desarrollo organizacional (significancia $=0.26$ ). La primera hipótesis específica es rechazada.

3. La hipótesis especifica dos postula que: El compromiso de los trabajadores incide directamente en el Desarrollo Organizacional en microempresas turísticas del valle del Mantaro. A mayor compromiso, mayor desarrollo, e inversamente. Con un nivel de confianza de $95 \%$ y significancia de $5 \%$ (0.000, el compromiso de los trabajadores, determina el desarrollo orga-

Páginas 81-85 
nizacional de las microempresas turísticas del valle del Mantaro. La hipótesis de investigación no se rechaza.

4. La hipótesis especifica tres postula que: La acción de los trabajadores incide en el Desarrollo Organizacional en microempresas turísticas del Valle del Mantaro de manera directa. A mayor acción de los trabajadores, mayor desarrollo.

Al respecto con nivel de confianza de $95 \%$ y significancia de $5 \%$ (0.000), la acción de los trabajadores, determina en el Desarrollo Organizacional en microempresas turísticas del valle del Mantaro de manera directa. Por consiguiente se acepta la hipótesis de investigación.

\section{REFERENCIAS}

Alcantara Napa, A. (2012). El talento humano y su influencia en el desarrollo organizacional de las microempresas de Grocio Prado, 2012. Chincha.

Chiavenato, I. (2009). Gesti\{on del Talento Humano. Mexico: Mc Graw Hill.

De Faria, F. A. (2004). Desarrollo organizacional: Enfoque integral. Máxico: Limusa.

Espinoza Montes, I. F., \& Espinoza Segovia, H. F. (2012). Competitividad de la micro y pequeña empresa turística del Valle del Mantaro. Huancayo: UNCP.

Gonzáles Espín, W. (2011). La gestión del talento humano y la motivación del personal en el Instituto Superior Tecnológico "Guaranda" en el primer semestre del 2011. Guaranda: Universidad Estatal de Bolívae.

Jericó, P. (2001). Gestión del talento. Madrid: Pearson. 